\title{
Retinal Tear, CTCAE
}

National Cancer Institute

\section{Source}

National Cancer Institute. Retinal Tear, CT CAE. NCI Thesaurus. Code C143814.

A disorder characterized by a small laceration of the retina, this occurs when the vitreous separates from the retina. Symptoms include flashes and floaters. 